SUBJECT AREAS:

NANOPARTICLES

SOLID-PHASE SYNTHESIS

Received

17 October 2014

Accepted

19 January 2015

Published

12 February 2015

Correspondence and requests for materials should be addressed to M.V.K.

(mvkovalenko@ethz.

ch) \section{Their Composites with Red Phosphorus as High-Performance Anode Materials for Na-ion Batteries}

\author{
Marc Walter ${ }^{1,2}$, Rolf Erni ${ }^{3}$ \& Maksym V. Kovalenko ${ }^{1,2}$ \\ 'Laboratory of Inorganic Chemistry, Department of Chemistry and Applied Biosciences, ETH Zürich, CH-8093 Zürich, Switzerland, \\ ${ }^{2}$ Laboratory for Thin Films and Photovoltaics, Empa - Swiss Federal Laboratories for Materials Science and Technology, CH-8600 \\ Dübendorf, Switzerland, ${ }^{3}$ Electron Microscopy Center, Empa - Swiss Federal Laboratories for Materials Science and Technology, \\ $\mathrm{CH}-8600$ Dübendorf, Switzerland.
}

Sodium-ion batteries increasingly become of immense research interest as a potential inexpensive alternative to Lithium-ion batteries. Development of high-energy-density negative electrodes (anodes) remains to be a great challenge, especially because of significant differences between lithium and sodium chemistries. Two Na-ion anode materials - antimony $(\mathrm{Sb})$ and phosphorus $(\mathrm{P})$ - have been recently shown to offer excellent cycling stability $(\mathrm{Sb})$ and highest known Na-ion charge storage capacity $(\mathrm{P})$. In this work we report on the synergistic $\mathrm{Na}$-ion storage in a $\mathrm{P} / \mathrm{Sb} / \mathrm{Cu}$-nanocomposite, produced by mixing inexpensive colloidal $\mathrm{Sb}$ nanocrystals with red $\mathrm{P}$ and with copper $(\mathrm{Cu})$ nanowires. In comparison to electrodes composed of only phosphorus, such $\mathrm{P} / \mathrm{Sb} / \mathrm{Cu}$-composite shows much greater cycling stability providing a capacity of

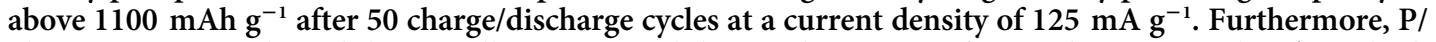
$\mathrm{Sb} / \mathrm{Cu}$-composite also exhibits excellent rate-capability, with capacity of more than $900 \mathrm{mAh} \mathrm{g}^{-1}$ at a high charge/discharge current density of $2000 \mathrm{~mA} \mathrm{~g}^{-1}$.

$\mathrm{R}$ echargeable batteries - which can store electricity of any origin in the form of chemical bond energy - are increasingly required to offer higher energy density, long cycle life and safety, all at sufficiently low cost of production. Sodium-ion batteries (SIBs) have long been neglected in favour of Lithium-ion batteries (LIBs), mainly due to the successful commercialization of LIBs early in 1990s. However, in light of the limited abundance and geographically uneven distribution of lithium salts (with main reserves in South America) concerns are raising regarding security of supply and cost of $\mathrm{Li}$ in the near future. Hence, for the growing need for batteries for electrical mobility and large-scale energy storage, conceptually identical SIBs are gaining attention as an economically viable alternative to $\mathrm{LIBs}^{1-4}$. While SIB cathodes have now reached similar performance to LIB cathodes ${ }^{5-12}$, the development of stable high-energy-density anode materials for SIBs still lacks well behind the Li-ion counterparts. In particular, silicon as well as graphite - which are two most successful LIB anode materials - have been reported to possess a negligible capacity for Na-ion storage ${ }^{13,14}$. Thus an extensive exploration of novel Na-ion anode materials has been launched in the recent years ${ }^{15-32}$.

Low cost and high Na-ion storage capacity are the major considerations for pre-selection of promising materials, whereas long cycle life has to be achieved via smart engineering of the electrodes at micro/nanoscale. In this regard, red phosphorus $(\mathrm{P})$ - the most chemically and thermodynamically stable allotrope of $\mathrm{P}$ - is both cheap and offers the highest known theoretical capacities for Na-ion storage $\left(2596 \mathrm{mAh} \mathrm{g}^{-1}\right.$ for $\mathrm{P} \leftrightarrow \mathrm{Na}_{3} \mathrm{P}$ transition). However, achieving such high capacities with acceptable cycling stability is hampered by massive increase of volume upon sodiation ( $\% \Delta V=291 \%)^{21}$ leading to the formation of cracks, loss of electrical contacts and fast capacity fading after several charge/discharge cycles. These difficulties are also commonplace for Si-based and other alloying anode materials in LIBs and are usually mitigated by nanostructuring of the active material ${ }^{33-51}$. Another common problem of $\mathrm{P}$ is the rather poor electronic conductivity and low diffusivity of $\mathrm{Na}^{+}$ions. Red $\mathrm{P}$ is a rather soft material with a low melting point and can be processed into a micro/nanostructured battery anode by hand or ball-milling with amorphous carbon nanoparticles (NPs) ${ }^{21-24}$. Such composites do show record-breaking near-theoretical capacities, but suffer from capacity fading due to both inherent instability of $\mathrm{P}$ and far from 
optimal performance of present-day $\mathrm{Na}$-ion electrolytes. The second most promising $\mathrm{Na}$-ion anode material is $\mathrm{Sb}$ (and Sb-based compounds), with theoretical capacity of $660 \mathrm{mAh} \mathrm{g}^{-1}$ (for $\mathrm{Sb} \leftrightarrow \mathrm{Na}_{3} \mathrm{Sb}$ conversion) and good cycling stability ${ }^{25-32,52}$. We recently introduced monodisperse Sb nanocrystals (NCs), produced via organometallic synthesis in nonpolar organic solvents, showing excellent cycling stability and rate capability (retention of $>80 \%$ of theoretical capacity at 20C-rate for charge and discharge, 1C-rate being current density of $\left.660 \mathrm{~mA} \mathrm{~g}^{-1}\right)^{53}$. Yet the fundamental drawback of organometallically prepared Sb NCs is the high cost of their synthesis and purification in organic solvents, using coordination compounds as precursors and expensive reducing agents.

In this study we thought to combine the best of $\mathrm{P}$ and $\mathrm{Sb}-$ much higher capacity of $\mathrm{P}$ and fast and stable cycling of $\mathrm{Sb}$ - into a functional synergy through the design of an inexpensive $\mathrm{P}-\mathrm{Sb}$ composite. We used the insights gained from our recent study on monodisperse $\mathrm{Sb} \mathrm{NCs}$; in particular, the fact that the performance of Sb NCs is only moderately size-dependent as long as NCs are in $20-100 \mathrm{~nm}$ size range. Hence, for the present study we developed a facile low-cost synthesis of Sb NCs, which, despite broader size distribution, exhibit even better performance than organometallically-synthesized $\mathrm{Sb}$ NCs. We were interested to find whether good electrochemical properties of Sb-P composite can be achieved without nanostructuring by high-energy mechanical milling, but simply by one-pot mixing the commercial red $\mathrm{P}$ with Sb NCs, solvent, binder and conductive additive. Further, through the addition of copper nanowires ( $\mathrm{Cu} \mathrm{NWs}$ ) we demonstrate the importance of tailoring the electronic conductivity and mechanic stability for enhancing electrochemical performance. The as-prepared $\mathrm{P} / \mathrm{Sb} / \mathrm{Cu}$-composite delivers a capacity of $>1300 \mathrm{mAh} \mathrm{g}^{-1}$ for 30 cycles corresponding to $>80 \%$ of its theoretical capacity. Moreover, the $\mathrm{P} / \mathrm{Sb} / \mathrm{Cu}$-composite shows outstanding rate capability retaining a capacity of $>900 \mathrm{mAh} \mathrm{g}^{-1}$ at a current of $2000 \mathrm{~mA} \mathrm{~g}^{-1}$.

\section{Results}

Inexpensive synthesis and electrochemical performance of $\mathbf{S b}$ NCs. Colloidal NCs make for an ideal battery material: they can be safely and conveniently produced and handled as stable dispersions in common solvents. Using monodisperse $\mathrm{Sb} \mathrm{NCs}$, we recently reported that $20 \mathrm{~nm}$ NCs exhibit higher Li-ion and Na-ion storage capacities and improved cycling stability, especially at higher rates of charge and discharge, than both smaller $\sim 10 \mathrm{~nm}$ particles and (sub)micron-sized $\mathrm{Sb}^{53}$. However, the synthesis of monodisperse Sb NCs involved expensive organic and coordination compounds (precursors, solvents, surfactants and reducing agents), as well as multiple steps of washing and removal of surface capping ligands. Yet a very important conclusion was obtained: mean particle size has a very modest effect on the electrochemical characteristics of Sb NCs, as long as it lies within the range of $20-100 \mathrm{~nm}$, in striking contrast to, for instance, Li-ion storage in Sn NCs, showing satisfactory performance only at sizes of $10 \mathrm{~nm}$ and below ${ }^{54}$. Such intrinsic tolerance of $\mathrm{Sb}$ to crystallite size has prompted us to search for an inexpensive synthetic route, even if the "precision" of the synthesis is somewhat sacrificed. For this work, we developed a much simpler and cheaper $(\sim 1 \mathrm{USD} / \mathrm{g})$ synthesis of Sb NCs via reduction of $\mathrm{SbCl}_{3}$ with $\mathrm{NaBH}_{4}$ at $60^{\circ} \mathrm{C}$ (for details, see the Experimental Section), yielding NCs of similar size as in a previous study, but at a cost that is lower by factor of $\sim 100$. The main features of this synthesis are the gram-scale and further up-scalable production with above $80 \%$ reaction yield, simple isolation of Sb NCs by centrifuging and convenient recycling of the solvent. The absence of surfactants obviates lengthy purification and surfactant-removal procedures. The only post-synthetic treatment is washing with water to remove the reaction byproducts (sodium chloride and borates). Figure 1 presents Sb NCs with the mean size of $\sim 20 \mathrm{~nm}$. The X-ray diffraction (XRD) pattern shows phase-pure, highly crystalline $\mathrm{Sb}$ NCs (space group N166, $R-3 \mathrm{~m}, \mathrm{a}=\mathrm{b}=0.4306 \mathrm{~nm}, \mathrm{c}=1.1288 \mathrm{~nm}$; calculated crystallite size of $22 \mathrm{~nm}$; see Supplementary Fig. S1 for Rietveld refinement), without any detectable traces of Sb oxides or reaction byproducts.

Preparation and electrochemical performance of Sb-P nanocomposites. Electrochemical performance of Sb NCs and of all other materials in this study has been tested in standard coin-type halfcells with metallic $\mathrm{Na}$ as counter electrode. Working electrodes were prepared by mixing Sb NCs (64 wt\%), with carbon black (CB) as conductive additive (21 wt\%) and carboxymethylcellulose (CMC, $15 \mathrm{wt} \%)$ as a water soluble polymeric binder, followed by doctorblade casting of thus formed slurry on $\mathrm{Cu}$ foil (mass loading $\sim 0.5 \mathrm{mg} / \mathrm{cm}^{2}$ ). $1 \mathrm{M} \mathrm{NaClO}_{4}$ in propylene carbonate was used as an electrolyte and fluoroethylenecarbonate (FEC) was used as electrolyte additive for stabilizing the solid-electrolyte interface (SEI ${ }^{46}$. In spite of the broader size distribution compared to organometallicallysynthesized Sb NCs, inexpensive Sb NCs developed in this study exhibit same or even better electrochemical characteristics (Fig. 2, for comparison see Supplementary Fig. S2).

Namely, near-theoretical capacity is obtained at $0.5-1 \mathrm{C}$ rates $(1 \mathrm{C}$ corresponds to current density of $660 \mathrm{~mA} \mathrm{~g}^{-1}$ ) without noticeable deterioration in the first 100 cycles. The small increase of the charge capacity observed in the charge/discharge curves might be due to the fact that not all of the electrode material is fully active during the first cycles due to kinetic limitations. Considering the massive volume changes for the sodiation/desodation reaction most likely the electrodes undergo restructuring in the first cycles leading to slightly better ionic and electronic conductivity, which explains the small increase of the charge capacity. Rate-capability tests indicate retention of at least $85 \%$ of capacity at a high rate of $20 \mathrm{C}\left(13.2 \mathrm{~A} \mathrm{~g}^{-1}\right)$ and
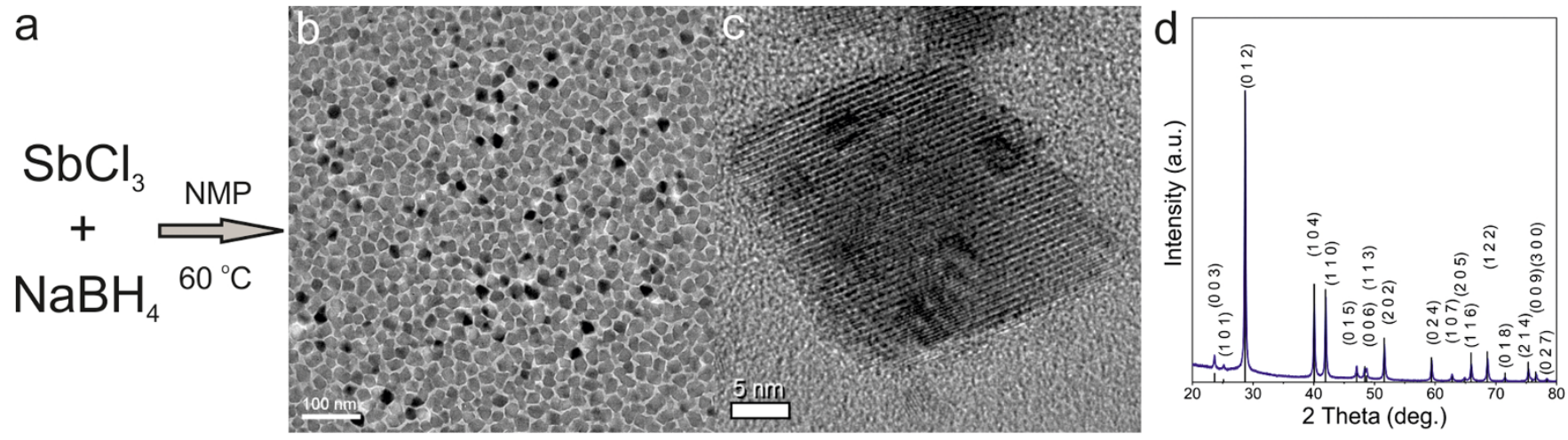

Figure 1 Synthesis and characterization of Sb NCs: (a) Reaction scheme; (b) TEM image; (c) high-resolution TEM image; (d) X-ray diffraction (XRD) pattern indexed to pure-phase hexagonal Sb (ICDD database, PDF Entry No.: 00-071-1173). 

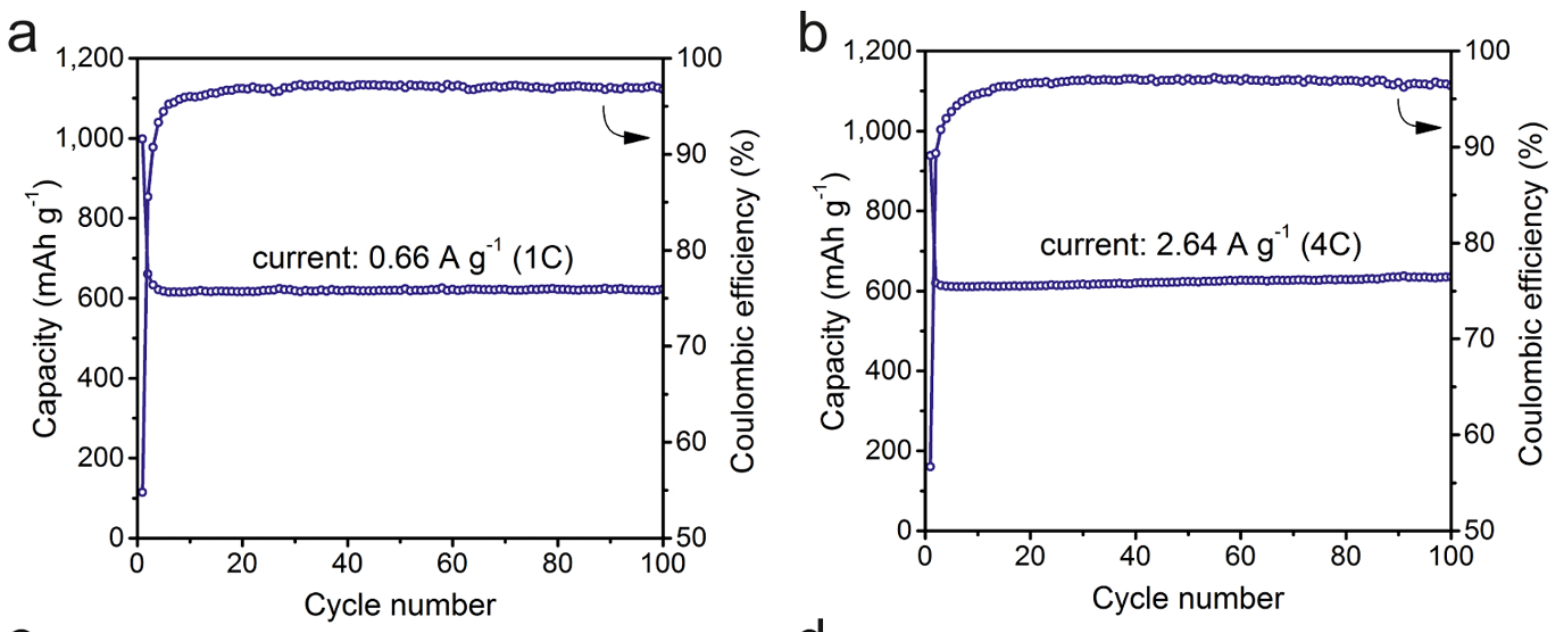

C

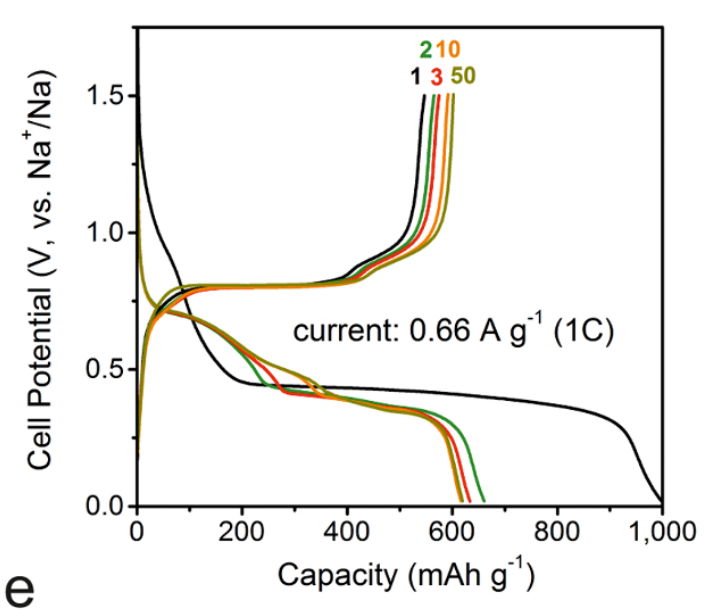

d
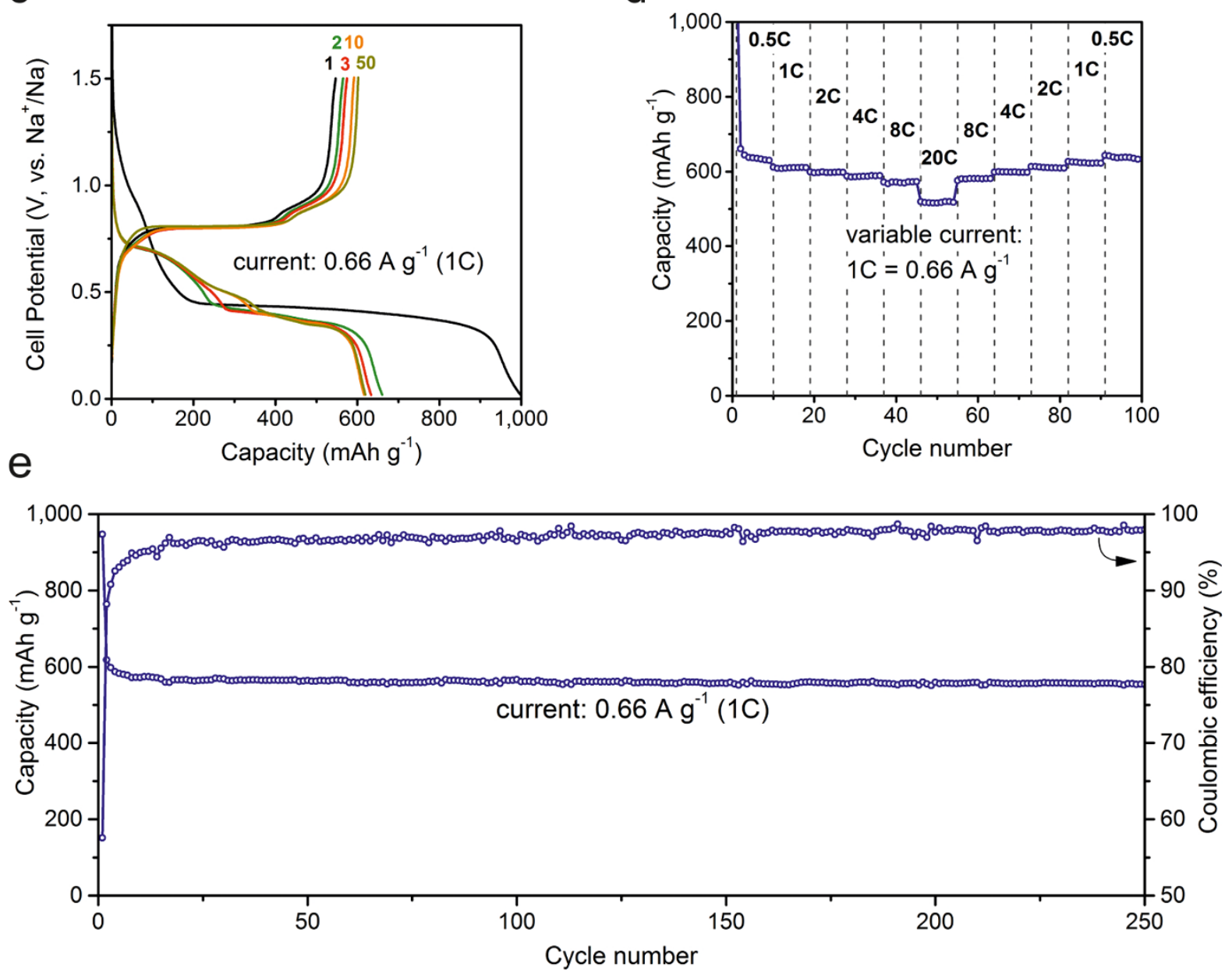

Figure 2 Electrochemical performance of $\sim 20 \mathrm{~nm} \mathrm{Sb} \mathrm{NCs} \mathrm{in} \mathrm{Na-ion} \mathrm{coin-type} \mathrm{half-cells:} \mathrm{(a)} \mathrm{and} \mathrm{(b)} \mathrm{illustrate} \mathrm{capacity} \mathrm{retention} \mathrm{at} \mathrm{a} \mathrm{rate} \mathrm{of} \mathrm{1C} \mathrm{and} \mathrm{4C}$ $\left(1 \mathrm{C}=0.66 \mathrm{Ag}^{-1}\right)$, respectively; (c) galvanostatic charge and discharge curves; (d) rate-capability tests (0.5-20C rate); (e) long-term cycling stability tests with enforced limitation of charge capacity to $550 \mathrm{mAh} \mathrm{g}^{-1}$. The composition of electrodes was Sb (64 wt\%), CB (21 wt\%) and CMC binder (15 wt \%). $1 \mathrm{M} \mathrm{NaClO}_{4}$ in propylene carbonate with addition fluoroethylene carbonate (10 wt $\%$, FEC) was used as electrolyte. All batteries were cycled in the $0.02-1.5 \mathrm{~V}$ potential range; capacities were normalized to the mass of active material.

full recovery of the capacity after decrease of the cycling rate to $0.5 \mathrm{C}$. The rate-capability of $\mathrm{Sb}$ is unprecedented for $\mathrm{Na}$-ion anodes, and is comparable to fastest Li-ion intercalation materials such as graphite $^{55}$, and Li titanates ${ }^{56}$. In addition, at a relatively high current density of $660 \mathrm{~mA} \mathrm{~g}^{-1}$ our Sb NC anodes show excellent cycling stability for at least 250 cycles, when the charge capacity is limited to $550 \mathrm{mAh} \mathrm{g}^{-1}$ (Fig. 2e). Similarly, if the charge capacity is limited to $400 \mathrm{mAh} \mathrm{g}^{-1} \mathrm{Sb}$ NCs retain their capacity for at least 400 cycles (Supplementary Fig. S3). Such impressively fast and stable operation of $\mathrm{Sb}$ anodes can be attributed to the synchronous effect of several properties. First, $\mathrm{Sb}$ is a good electronic conductor due to its semimetallic nature. Second, its crystalline structure is characterized by the low atomic packing factor of just $39 \%{ }^{57}$ and the crystal structure is comprised of puckered layered planes with large channels for 
a
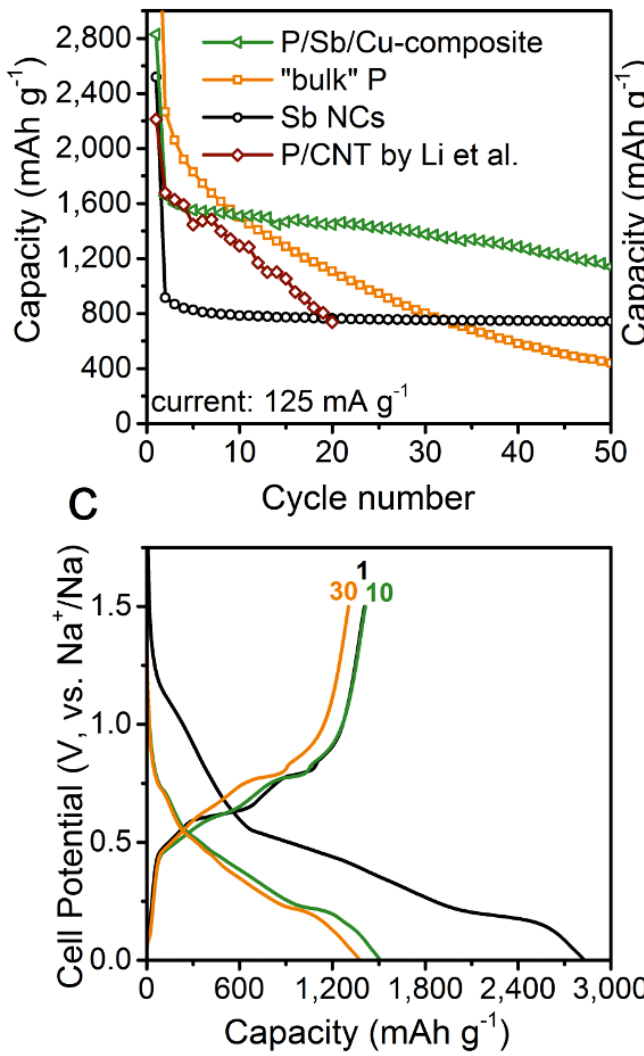

b
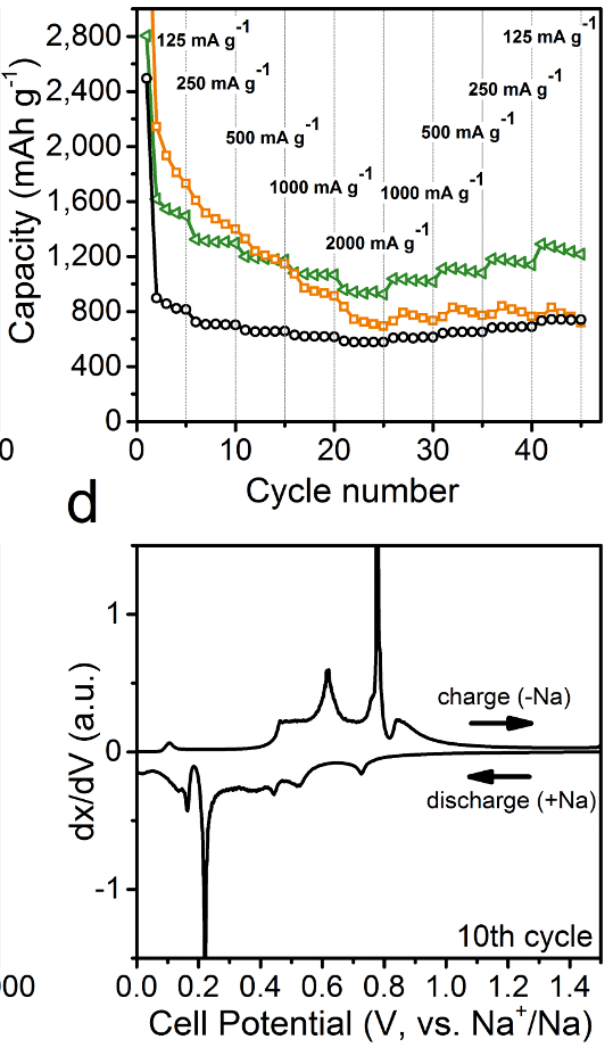

Figure 3 Electrochemical performance of the $\mathrm{P} / \mathrm{Sb} / \mathrm{Cu}$ composite electrodes in comparison to $\mathrm{Sb} \mathrm{NCs}$ and red $\mathrm{P}$ : (a) cycling stability at a current density of $125 \mathrm{~mA} \mathrm{~g}^{-1}$; (b) rate-capability tests; (c) galvanostatic charge and discharge curves; (d) differential capacity plot for the $10^{\text {th }}$ cycle. The data of $\mathrm{Li}$ et al. for a hand-milled composite of red $\mathrm{P}$ and carbon nanotubes, tested under similar cycling conditions, are shown for comparison ${ }^{24}$. All electrodes were formulated as (active-material) $: \mathrm{CB}: \mathrm{CMC}=40 \%: 40 \%: 20 \%$ or, for samples containing Cu NWs, (active material) $: \mathrm{CB}: \mathrm{Cu}: \mathrm{CMC}=40 \%: 30 \%$ : $10 \%: 20 \%$ by weight and cycled in the $0-1.5 \mathrm{~V}$ potential range.

diffusion of alkali ions. Insertion of $\mathrm{Na}$-ions into $\mathrm{Sb}$ involves only one crystalline $\left(\mathrm{Na}_{3} \mathrm{Sb}\right)$ and several amorphous phases, including amorphous $\mathrm{Sb}^{52}$. De-insertion primarily occurs as direct $\mathrm{Na}_{3} \mathrm{Sb}_{\text {hex }} \rightarrow \mathrm{Sb}_{\text {amorphous }}$ transition. The reduced number of intermediate crystalline phases greatly enhances the conversion kinetics. The theoretical value for volumetric expansion upon full sodiation to hexagonal $\mathrm{Na}_{3} \mathrm{Sb}$ can be estimated from the difference in the molar volumes $\left(\% V_{m}\right)$ between the final $\left(\mathrm{Na}_{3} \mathrm{Sb}\right)$ and the initial metallic $(S b)$ phases: $\% \Delta V=100 \% \times\left[V_{m}\left(N a_{x} S b\right)-V_{m}(S b)\right] / V_{m}(S b)=$ $290 \%$. It is the effect of isotropic, nearly strain-free expansion/contraction of amorphous phases that can explain the tolerance of $\mathrm{Sb}$ to such drastic volumetric changes.

Fundamentally, the formation of composites opens up multiple new opportunities for tuning the electronic and ionic transport, surface chemistry, porosity and mean particle size, with strong implications for rate capability and cycling stability. The initial goal of this study was to obtain a compelling combination of the two materials: functional synergy of higher capacity of $\mathrm{P}$ with high-rate capability and high cyclability of Sb.

P undergoes very similar to Sb volumetric changes of 290\% upon full sodiation to $\mathrm{Na}_{3} \mathrm{P}^{23}$. At the same time, $\mathrm{P}$ shows much faster capacity fade $\mathrm{e}^{21-23}$, which can be attributed to poor electronic and ionic conductivity. Replacing some of the $\mathrm{P}$ with Sb NCs might give rise to improved electrochemical properties as (sub)micron-sized $\mathrm{P}$ particles are more diluted in a conductive nanoscopic matrix of $\mathrm{Sb}$ and $\mathrm{CB}$. For $1: 1$ mixture of $\mathrm{Sb}$ and $\mathrm{P}$, one can expect capacities up to theoretical weighed average of $1628 \mathrm{mAh}^{-1}$.

Figure 3 captures the main result of this communication - significant enhancement in reversibility of $\mathrm{Na}$-ion storage by combining $\mathrm{Sb}$
NCs, "bulk" $\mathrm{P}$ and $\mathrm{Cu}$ NWs. Electrodes containing either Sb NCs, commercial red $\mathrm{P}$ or $1: 1$ mixtures thereof, with or without $\mathrm{Cu}$ NWs, were prepared with an overall mass ratio active-material: $\mathrm{CB}$ : $\mathrm{CMC}$ $=40 \%: 40 \%: 20 \%$. Such relatively high fraction of conductive carbons such as $\mathrm{CB}$ are generally used for $\mathrm{P}$-based electrodes in $\mathrm{Na}$-ion batteries to provide sufficient electronic conductivity and mechanical stability of the electrodes ${ }^{21-24}$. Currents and capacities were related to the active mass without CB. From TEM and SEM images can be seen that the size of "bulk" $P$ before and after mechanical mixing is retained (see Fig. 4 and Supplementary Fig. S4). As shown in Fig. 3, electrodes comprising $40 \%$ of pure Sb NCs deliver stable capacities and excellent rate capability. The fact, that at a current of $125 \mathrm{~mA} \mathrm{~g}^{-1}$ the apparent capacity of Sb NCs exceeds the theoretical value of $660 \mathrm{mAh} \mathrm{g}^{-1}$, can be explained by the contribution from CB (see Supplementary Fig. S5). At this current density, CB exhibits stable capacity of ca. $100 \mathrm{mAh} \mathrm{g}^{-1}$, and thus may be contributing $\sim 100 \mathrm{mAh}^{-1}$ in its 1:1 mixture with $\mathrm{Sb}$ (or $\mathrm{Sb} / \mathrm{P}$ ). Electrodes composed of "bulk" $\mathrm{P}$ do show theoretical capacities in the first cycles, indicating that electronic connectivity is satisfactory in the as-prepared electrode, but undergo severe capacity fading to $440 \mathrm{mAh} \mathrm{g}^{-1}$ after 50 cycles. This result is fully consistent with the available literature on mechanically produced $\mathrm{P} /$ carbon electro$\operatorname{des}^{23,24}$. For instance, Fig. 3a shows for comparison also the data of $\mathrm{Li}$ et al. for P/carbon nanotube (CNT)-composite, measured under similar conditions as in our work ${ }^{24}$. Experiments using $1: 1$ mixtures of "bulk" P and Sb NCs indicate electrochemical performance which exceeds the mathematic sum of the individual contributions, leading to retention of more than $800 \mathrm{mAh} \mathrm{g}^{-1}$ of capacity after 50 cycles (Supplementary Fig. S6). This improved capacity retention observed 
for electrodes of $\mathrm{Sb} / \mathrm{P}$ electrodes can be explained by the lower content of $\mathrm{P}$. Due to the higher "dilution " of $\mathrm{P}$ particles in a matrix of $\mathrm{Sb}$ $\mathrm{NCs}$ and $\mathrm{CB}$ the volume changes can be buffered more effectively and electrodes are suffering from less mechanical stress leading to better cycling stability. Further major improvement has been attained by replacing some $\mathrm{CB}$ with $\mathrm{Cu}$ NWs. Cu NWs were synthesized according to a previously published procedure by Guo et al. (Supplementary Fig. S7 $)^{58}$. We note that Cu NWs are becoming comparably inexpensive. For instance, during the preparation of this manuscript, Li et al. reported facile synthesis of Cu NWs at a cost of $4.20 \mathrm{USD} / \mathrm{g}^{59}$. Further, it is generally well known that the cost of synthesis may further go down by up to an order of magnitude when material is produced on industrial scale. Electrodes comprising 1:1 mixtures of "bulk" P/Sb NCs with 10 wt\% Cu NWs (i.e. 25\% of CB was replaced with $\mathrm{Cu} \mathrm{NWs}$ ) delivered capacity of $>1300 \mathrm{mAh} \mathrm{g}^{-1}$ for 30 cycles, corresponding to $>80 \%$ of the theoretical capacity for this mixture $\left(1628 \mathrm{mAh} \mathrm{g}^{-1}\right)$. After 50 cycles still a capacity of $>1100 \mathrm{mAh} \mathrm{g}^{-1}$ is retained, by $60 \%$ higher than in $\mathrm{Cu}$-free samples and almost three times the value for "bulk" $\mathrm{P}$, clearly manifesting the synergy between $\mathrm{P}$ and nanoscopic $\mathrm{Sb}$ and $\mathrm{Cu}$.

An obvious benefit of the $\mathrm{P} / \mathrm{Sb} / \mathrm{Cu}$ formulation is seen also in the rate-capability tests (Fig. 3c). For comparison, all formulations of electrodes were subjected first to stepwise increase of the current density to $2000 \mathrm{~mA} \mathrm{~g}^{-1}$, and then to stepwise reduction of the current. Whereas electrodes of "bulk" $P$ fail to recover their capacity, the $\mathrm{P} / \mathrm{Sb} / \mathrm{Cu}$-composite is able to retain the same capacity level as after the continuous cycling at $125 \mathrm{~mA} \mathrm{~g}^{-1}$ (Fig. 3a). In addition, the $\mathrm{P} / \mathrm{Sb} /$ $\mathrm{Cu}$-composite shows excellent rate-capability with capacities of $>900 \mathrm{mAh} \mathrm{g}^{-1}$ at $2000 \mathrm{~mA} \mathrm{~g}^{-1}$ current density.

An advantage of $\mathrm{P}$ is its lower electrode potential compared to $\mathrm{Sb}$, potentially giving rise to higher energy density - that is higher voltage $\times$ capacity product of a full cell battery - when combined with high-voltage cathode. Figure $3 \mathrm{c}$ presents galvanostatic charge (desodiation) and discharge (sodiation) curves for $\mathrm{P} / \mathrm{Sb} / \mathrm{Cu}$-composite, showing that sodiation and desodiation occurs at an average voltage of $0.5 \mathrm{~V}$, highly suitable for anode applications. Additional hints can be found from differential capacitance plots (dQ/dV, Fig. $3 \mathrm{~d}$ ), derived from the charge/discharge curves, presented for the $10^{\text {th }}$ cycle (within the regime of stable cycling). In the first cycle (not shown here), two desodiation features at 0.6 and $0.8 \mathrm{~V}$ indicate individual contributions from $\mathrm{P}$ and $\mathrm{Sb}$, as observed in reference electrodes comprising red $\mathrm{P}$ and Sb NCs (see Supplementary Fig. S8). However, in the subsequent cycles an additional broad component in 0.4-0.8 V range is built up, not present in $\mathrm{Cu}$-free $\mathrm{P} / \mathrm{Sb}$ electrodes.
Further, we have observed that $\mathrm{P} / \mathrm{Sb}$ electrodes exhibit an additional sodiation process at $0.16 \mathrm{~V}$, not seen for electrodes composed of only $\mathrm{P}$ or Sb (see Supplementary Fig. S8). All other processes correspond to the individual contributions from $\mathrm{P}$ and $\mathrm{Sb}^{23,52}$. Temporal separation of electrochemical processes due to the spread of sodiation (desodiation) features over the broad voltage range may in fact have a stabilizing effect. In our recent study on Sn-Ge nanocomposites as LIB anodes ${ }^{60}$, as well as in numerous other reports on LIBs, stepwise lithiation and delithiation in composites of several electrode materials has been shown to enhance the mechanical stability, as compared to instant expansion of the whole electrode.

The coulombic efficiency - the ratio between the amounts of electrical charge spent for sodiation and desodiation processes in each cycle - provides an important insight into the reversibility of the charging/discharging processes. Due to the formation of the solidelectrolyte interface (SEI) the coulombic efficiency for the first cycle with $\mathrm{P} / \mathrm{Sb} / \mathrm{Cu}$ electrodes is only $60 \%$, but reached $95 \%$ in the subsequent cycles at a current of $125 \mathrm{~mA} \mathrm{~g}^{-1}$. This initially low coulombic efficiency can be explained by the small size of Sb NCs and $\mathrm{Cu}$ NWs, which provides a large surface area for the irreversible electrolyte decomposition during the first discharge. During cycling at a relatively high current of $2000 \mathrm{~mA} \mathrm{~g}^{-1}$ the coulombic efficiency increased to $98 \%$. As commonly reported for conversion anode materials with large volume work during cycling, the cracking of SEI layer leads to continuous decomposition of the electrolyte on the freshly exposed surfaces. This constant reformation of the SEI lowers the coulombic efficiency and long-term cycling stability due to degradation of both the electrodes and electrolytes.

\section{Discussion}

Figure 4 depicts the stabilizing effect present in $\mathrm{P} / \mathrm{Sb} / \mathrm{Cu}$ electrodes. $\mathrm{Cu} \mathrm{NWs}$ are assumed to cause better mechanic stability and improved electronic connectivity, slowing down the loss of electric contact due to pulverization of $\mathrm{P}$ particles. Cu NWs can trap fractured material to a degree that CB particles cannot provide. Overall, dilution of the (sub)micron-sized $\mathrm{P}$ particles, when mixed with $\mathrm{Sb}$ NCs and $\mathrm{Cu}$ NWs, most likely improves the overall mechanical stability of the electrode leading to retarded cracking and crumbling during cycling. High-angle angular dark field-scanning transmission electron images (HAADF-STEM, Fig. 4b), bright-field STEM images (Supplementary Fig. S9), elemental mapping by energy-dispersive Xray spectrometry (EDX-map, Supplementary Fig. S9) and scanning electron microscopy images (SEM) clearly evidence intimate intermixing in $\mathrm{P} / \mathrm{Sb} / \mathrm{Cu}$ electrodes. We thus attribute the herein presented a
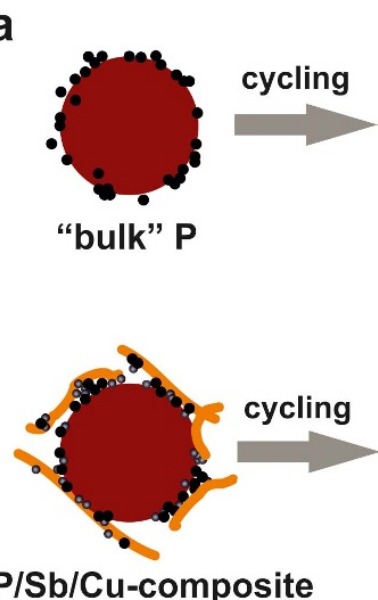

$\mathrm{P} / \mathrm{Sb} / \mathrm{Cu}$-composite
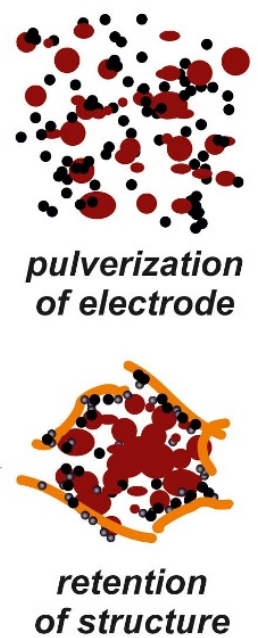

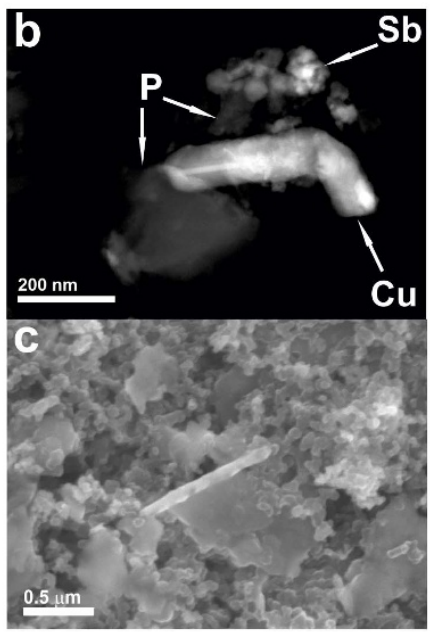

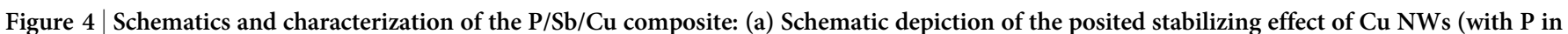

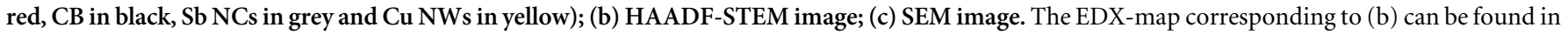
the Supporting Information. 
outstanding electrochemical properties of the $\mathrm{P} / \mathrm{Sb} / \mathrm{Cu}$-composite to the effective embedding of "bulk" $\mathrm{P}$ in a $\mathrm{Sb} / \mathrm{Cu} / \mathrm{CB}$ matrix.

It is important to point out that of all measured electrodes only the ones comprising "bulk" $\mathrm{P}$ and Sb NCs with 10 wt\% Cu NWs had clearly emerged as champion devices. Replacing Sb NCs with micrometre-sized commercial Sb led to significantly poorer performance (see Supplementary Fig. S10), very similar to purely P-based electrodes. Similarly, whereas electrodes containing both "bulk $\mathrm{P}$ " and $\mathrm{Cu}$ NWs exhibit higher cycling stability than in the absence of Cu NWs, they fall well behind the $\mathrm{P} / \mathrm{Sb} / \mathrm{Cu}$-composite (see Supplementary Fig. $\mathrm{S} 11$ ). In addition, increasing the content of $\mathrm{Cu}$ NWs from 10 to $20 \mathrm{wt} \%$ did not improve the cycling stability further, but rather led to lower capacities (Supplementary Fig. S6). Considering that electrodes are composed of particles with very different size, this observation indicates that in order to obtain the optimal electrical contact in the electrodes and therefore highest capacity the ratio between $\mathrm{CB}$ particles and $\mathrm{Cu}$ NWs needs to be properly balanced. Finally, SEM images after extended galvanostatic cycling (Supplementary Fig. S12, 100 cycles) indicate that $\mathrm{P}$ particles become smaller, some cracks develop, but overall mechanical integrity of the electrodes is retained.

A great deal of work still has to be focused on improving the longterm cycling stability of the electrodes. Besides the effect of the active storage material, the cycling stability is a complex function of the electrode formulation (chemistry and amounts of binder and conductive additive), porosity, electrode thickness, electrolyte, temperature etc. In particular, coulombic efficiencies of $\sim 92 \%, \sim 95 \%$, and 97-98\% for $\mathrm{P}, \mathrm{P} / \mathrm{Sb} / \mathrm{Cu}$ and $\mathrm{Sb}$ electrodes respectively, indicate a continuous consumption of electrolyte for side anodic reactions reformation of unstable SEI layer after each cycle and, very likely, reactivity of $\mathrm{Na}_{3} \mathrm{P}$ towards the electrolyte ${ }^{23}$. Smart engineering of the electrodes for instance by designing secondary structures combined with judicious choice of electrolytes and electrolyte additives will enable higher stability of SEI layer in future studies. We note that in the field of SIBs the problems of optimizing the chemistry of electrolytes and understanding of SEI formation had so far received much less attention as compared to 30 years of research on LIBs, and the knowledge are not necessarily interchangeable between these two fields.

In summary, this work showcases a strategy for constructing a composite $\mathrm{Na}$-ion anode material, which combines several active materials with functional synergy between them. First, we presented a facile low-cost synthesis of Sb NCs that exhibit outstanding highrate capability and long cycling life as anode material in SIBs. Compared to previous syntheses of $\mathrm{Sb}$ nanostructures by us and by others $^{53,61-63}$, the advantages are the use of inexpensive reagents, the absence of any surfactants, simple washing procedure, high reaction yield and scalability. In fact, all that needs to be done for the synthesis of $\mathrm{Sb} \mathrm{NCs}$ is addition of the $\mathrm{Sb}$ precursor solution at very moderate temperature $\left(60^{\circ} \mathrm{C}\right)$ to the solution of $\mathrm{NaBH}_{4}$, which can easily be carried out on the industrial scale with either a batch or a continuous flow reactor. Purification of these $\mathrm{Sb}$ NCs involves only rinsing with water and filtering or centrifugation. A compelling $\mathrm{P} / \mathrm{Sb} / \mathrm{Cu}$ composite anode was then devised by simple mechanic mixing of Sb NCs with "bulk" red P and Cu NWs. In this composite, larger (sub)micron-sized $\mathrm{P}$ particles are embedded into a nanoscopic matrix of intimately intermixed Sb NCs, Cu NWs and nanoparticulate CB. Electrodes composed of $1: 1$ mixtures of "bulk" P/Sb NCs with $10 \mathrm{wt} \% \mathrm{Cu}$ NWs delivered charge storage capacities of $>1300 \mathrm{mAh} \mathrm{g}^{-1}$ for 30 cycles and $>1100 \mathrm{mAh} \mathrm{g}^{-1}$ after 50 cycles at a current density of $125 \mathrm{~mA}$ $\mathrm{g}^{-1}$. Furthermore, reversible capacities of $>900 \mathrm{mAh} \mathrm{g}^{-1}$ at high current density of $2000 \mathrm{~mA} \mathrm{~g}^{-1}$ have been obtained as well.

\section{Methods}

Synthesis of Sb NCs and Cu NWs. To synthesize $\sim 20 \mathrm{~nm} \mathrm{Sb} \mathrm{NCs,} \mathrm{NaBH}_{4}$ (48 mmol, 98\%, ABCR) was dissolved in distilled N-Methyl-2-pyrrolidone (NMP, $51 \mathrm{~mL}, 99.8 \%$, Fluorochem Ltd) in a three-necked flask under nitrogen and heated to $60^{\circ} \mathrm{C} . \mathrm{SbCl}_{3}(12 \mathrm{mmol}, 99 \%, \mathrm{ABCR})$ dissolved in NMP $(9 \mathrm{~mL})$ were quickly injected via syringe. The reaction mixture instantly turned black and was cooled down immediately using a water-ice bath. After cooling to room-temperature, Sb NCs have been separated from the solution by centrifugation $(8000 \mathrm{rpm}, 4 \mathrm{~min})$ and washed three times with deionized water $(30 \mathrm{~mL})$ to remove unreacted $\mathrm{NaBH}_{4}$ and watersoluble side products such as $\mathrm{NaCl}$. The reaction product was finally dried in the vacuum oven at room temperature, yielding $1.2 \mathrm{~g}$ of Sb NCs (82\% reaction yield). NMP solvent recovered after centrifuging can be reused with or without distillation, giving very similar results. Cu NWs were synthesized according to a procedure published by Guo et al. ${ }^{58}$

Electrode fabrication, cell assembly and electrochemical measurements. The following battery components were used: carbon black (Super C65, TIMCAL), carboxymethyl cellulose (CMC, Grade: 2200, Lot No. B1118282, Daicel Fine Chem Ltd.), $\mathrm{NaClO}_{4}$ (98\%, Alfa Aesar, additionally dried), propylene carbonate (BASF, battery grade), 4-fluoro-1,3-dioxolan-2-one (FEC, Hisunny Chemical, battery grade), glass microfiber separator (GF/D, Cat No.1823-257, Whatman), and Cu foil (9 $\mu \mathrm{m}$, MTI Corporation).

In a typical electrode preparation, the respective materials were combined with deionized water and mixed in a Fritsch Pulverisette 7 classic planetary mill for $1 \mathrm{~h}$ at $500 \mathrm{rpm}$. Mixing weight ratios were $\mathrm{Sb}: \mathrm{CB}: \mathrm{CMC}=64 \%: 21 \%: 15 \%$ for pure $\mathrm{Sb}$ NCs (Fig. 2), and P/Sb:CB :CMC $=40 \%: 40 \%: 20 \%$ or $\mathrm{P} / \mathrm{Sb}: \mathrm{CB}: \mathrm{Cu}: \mathrm{CMC}=$ $40 \%: 30 \%: 10 \%: 20 \%$ (Fig. 3). The aqueous slurries were coated onto Cu current collectors and then dried overnight at $80^{\circ} \mathrm{C}$ under vacuum prior to use. All electrochemical measurements were conducted in homemade, reusable and air-tight cointype cells assembled in an Ar-filled glove box $\left(\mathrm{O}_{2}<1 \mathrm{ppm}, \mathrm{H}_{2} \mathrm{O}<1 \mathrm{ppm}\right)$. Elemental sodium was employed as both reference and counter electrode. As electrolyte $1 \mathrm{M} \mathrm{NaClO}_{4}$ in propylene carbonate with $10 \%$ fluoroethylene carbonate was used. Glass fiber was used as separator. Galvanostatic cycling tests were carried out at room temperature on MPG2 multi-channel workstation (BioLogic). Capacities were normalized by the mass of active material.

Materials characterization. Transmission Electron Microscopy (TEM) images were obtained with a Philips CM30 TEM microscope at $300 \mathrm{kV}$ using carbon-coated Cu grids as substrates (Ted-Pella). Scanning transmission electron microscopy (STEM) and EDX mapping were performed on a JEOL 2200FS TEM/STEM microscope. Scanning electron microscopy (SEM) was performed using a NanoSEM 230. Powder $\mathrm{X}$-ray diffraction (XRD) was measured on a STOE STADI P powder X-ray diffractometer.

1. Van Noorden, R. The rechargeable revolution: A better battery. Nature 507, 26-28 (2014).

2. Palomares, V. et al. Na-ion batteries, recent advances and present challenges to become low cost energy storage systems. Energy Environ. Sci. 5, 5884-5901 (2012).

3. Slater, M. D., Kim, D., Lee, E. \& Johnson, C. S. Sodium-ion batteries. Angew. Chem. Int. Ed. 23, 947-958 (2013).

4. Pan, H., Hu, Y.-S. \& Chen, L. Room-temperature stationary sodium-ion batteries for large-scale electric energy storage. Energy Environ. Sci. 6, 2338-2360 (2013).

5. Park, Y. U. et al. A new high-energy cathode for a Na-ion battery with ultrahigh stability. J. Am. Chem. Soc. 135, 13870-13878 (2013).

6. Yuan, D. et al. Synthesis and electrochemical behaviors of layered $\mathrm{Na} 0.67[\mathrm{Mn} 0.65 \mathrm{Co} 0.2 \mathrm{Ni} 0.15] \mathrm{O} 2$ microflakes as a stable cathode material for sodium-ion batteries. J. Mater. Chem. A 1, 3895-3899 (2013).

7. You, Y., Wu, X.-L., Yin, Y.-X. \& Guo, Y.-G. High-quality Prussian blue crystals as superior cathode materials for room-temperature sodium-ion batteries. Energy Environ. Sci. 7, 1643-1647 (2014)

8. Buchholz, D., Chagas, L. G., Winter, M. \& Passerini, S. P2-type layered $\mathrm{Na} 0.45 \mathrm{Ni} 0.22 \mathrm{Co} 0.11 \mathrm{Mn} 0.66 \mathrm{O} 2$ as intercalation host material for lithium and sodium batteries. Electrochim. Acta 110, 208-213 (2013).

9. Buchholz, D. et al. Toward Na-ion batteries-synthesis and characterization of a novel high capacity $\mathrm{Na}$ ion intercalation material. Chem. Mater. 25, 142-148 (2012).

10. Yabuuchi, N. et al. P2-type $\mathrm{Nax}[\mathrm{Fe} 1 / 2 \mathrm{Mn} 1 / 2] \mathrm{O} 2$ made from earth-abundant elements for rechargeable Na batteries. Nat. Mater. 11, 512-517 (2012).

11. Huang, W. et al. Detailed investigation of $\mathrm{Na} 2.24 \mathrm{FePO} 4 \mathrm{CO} 3$ as a cathode material for Na-ion batteries. Sci. Rep. 4, 4188; DOI: $4110.1038 /$ srep04188 (2014).

12. Lu, Y. H., Wang, L., Cheng, J. G. \& Goodenough, J. B. Prussian blue: a new framework of electrode materials for sodium batteries. Chem. Commun. 48, 6544-6546 (2012).

13. Ge, P. Electrochemical intercalation of sodium in graphite. Solid State Ionics $\mathbf{2 8 -}$ 30, 1172-1175 (1988)

14. Komaba, S. et al. Redox reaction of Sn-polyacrylate electrodes in aprotic Na cell. Electrochem. Commun. 21, 65-68 (2012).

15. Wen, Y. et al. Expanded graphite as superior anode for sodium-ion batteries. Nat. Commun. 5, 4033 (2014).

16. Wu, L. M., Buchholz, D., Bresser, D., Chagas, L. G. \& Passerini, S. Anatase TiO2 nanoparticles for high power sodium-ion anodes. J. Power Sources 251, 379-385 (2014).

17. Yu, D. Y. et al. High-capacity antimony sulphide nanoparticle-decorated graphene composite as anode for sodium-ion batteries. Nat. Commun. 4, 2922 (2013). 
18. Wang, Y. et al. A zero-strain layered metal oxide as the negative electrode for longlife sodium-ion batteries. Nat. Commun. 4, 2365 (2013).

19. Sun, Y. et al. Direct atomic-scale confirmation of three-phase storage mechanism in $\mathrm{Li}(4) \mathrm{Ti}(5) \mathrm{O}(1)(2)$ anodes for room-temperature sodium-ion batteries. Nat. Commun. 4, 1870 (2013).

20. Lin, Y. M. et al. Sn-Cu nanocomposite anodes for rechargeable sodium-ion batteries. ACS Appl. Mater. Interfaces 5, 8273-8277 (2013).

21. Qian, J., Wu, X., Cao, Y., Ai, X. \& Yang, H. High capacity and rate capability of amorphous phosphorus for sodium ion batteries. Angew. Chem. Int. Ed. 52, 4633-4636 (2013)

22. Kim, Y. et al. An amorphous red phosphorus/carbon vomposite as a promising anode material for sodium ion batteries. Adv. Mater. 25, 3045-3049 (2013).

23. Yabuuchi, N. et al. Phosphorus electrodes in sodium cells: small volume expansion by sodiation and the surface-stabilization mechanism in aprotic solvent. ChemElectroChem 1, 580-589 (2014).

24. Li, W.-J., Chou, S.-L., Wang, J.-Z., Liu, H.-K. \& Dou, S.-X. Simply mixed commercial red phosphorus and carbon nanotube composite with exceptionally reversible sodium-ion storage. Nano Lett. 13, 5480-5484 (2013).

25. Xiao, L. et al. High capacity, reversible alloying reactions in $\mathrm{SnSb} / \mathrm{C}$ nanocomposites for Na-ion battery applications. Chem. Commun. 48, 3321-3323 (2012).

26. Darwiche, A., Sougrati, M. T., Fraisse, B., Stievano, L. \& Monconduit, L. Facile synthesis and long cycle life of $\mathrm{SnSb}$ as negative electrode material for $\mathrm{Na}$-ion batteries. Electrochem. Commun. 32, 18-21 (2013).

27. Zhu, Y. et al. Electrospun Sb/C Fibers for a Stable and Fast Sodium-Ion Battery Anode. ACS Nano 7, 6378-6386 (2013).

28. Zhou, X., Dai, Z., Bao, J. \& Guo, Y.-G. Wet milled synthesis of an Sb/MWCNT nanocomposite for improved sodium storage. J. Mater. Chem. A 1, 13727-13731 (2013)

29. Nam, D. H., Hong, K. S., Lim, S. J. \& Kwon, H. S. Electrochemical synthesis of a three-dimensional porous $\mathrm{Sb} / \mathrm{Cu} 2 \mathrm{Sb}$ anode for Na-ion batteries. J. Power Sources 247, 423-427 (2014).

30. Wu, L. et al. Sb-C nanofibers with long cycle life as an anode material for highperformance sodium-ion batteries. Energy Environ. Sci. 7, 323-328 (2014).

31. Farbod, B. et al. Anodes for Sodium Ion Batteries Based on Tin-GermaniumAntimony Alloys. ACS Nano 8, 4415-4429 (2014).

32. Nithya, C. \& Gopukumar, S. rGO/nano Sb composite: a high performance anode material for $\mathrm{Na}+$ ion batteries and evidence for the formation of nanoribbons from the nano rGO sheet during galvanostatic cycling. J. Mater. Chem. A 2, 10516-10525 (2014).

33. Goodenough, J. B. \& Kim, Y. Challenges for rechargeable Li batteries. Chem. Mater. 22, 587-603 (2009).

34. Liu, N. et al. A pomegranate-inspired nanoscale design for large-volume-change lithium battery anodes. Nat. Nanotechnol. 9, 187-192 (2014).

35. Nitta, N. \& Yushin, G. High-Capacity Anode Materials for Lithium- Ion Batteries: Choice of Elements and Structures for Active Particles. Part. Part. Syst. Charact. 31, 317-336 (2014)

36. Chan, C. K. et al. High-performance lithium battery anodes using silicon nanowires. Nat. Nanotechnol. 3, 31-35 (2008).

37. Hu, Y.-S. et al. Superior Storage Performance of a Si@SiOx/C Nanocomposite as Anode Material for Lithium-Ion Batteries. Angew. Chem. Int. Ed. 47, 1645-1649 (2008)

38. Magasinski, A. et al. High-performance lithium-ion anodes using a hierarchical bottom-up approach. Nat. Mater. 9, 353-358 (2010).

39. Yao, Y. et al. Interconnected Silicon Hollow Nanospheres for Lithium-Ion Battery Anodes with Long Cycle Life. Nano Lett. 11, 2949-2954 (2011).

40. Chan, C. K., Patel, R. N., O'Connell, M. J., Korgel, B. A. \& Cui, Y. Solution-Grown Silicon Nanowires for Lithium-Ion Battery Anodes. ACS Nano 4, 1443-1450 (2010)

41. Wu, H. et al. Engineering Empty Space between Si Nanoparticles for Lithium-Ion Battery Anodes. Nano Lett. 12, 904-909 (2012).

42. Bruce, P. G., Scrosati, B. \& Tarascon, J.-M. Nanomaterials for Rechargeable Lithium Batteries. Angew. Chem. Int. Ed. 47, 2930-2946 (2008).

43. Chan, C. K., Zhang, X. F. \& Cui, Y. High Capacity Li Ion Battery Anodes Using Ge Nanowires. Nano Lett. 8, 307-309 (2007).

44. Park, M.-H. et al. Silicon Nanotube Battery Anodes. Nano Lett. 9, 3844-3847 (2009)

45. Bogart, T. D. et al. Lithium Ion Battery Peformance of Silicon Nanowires with Carbon Skin. ACS Nano 8, 915-922 (2013).

46. Chockla, A. M., Klavetter, K. C., Mullins, C. B. \& Korgel, B. A. Tin-seeded silicon nanowires for high capacity Li-ion batteries. Chem. Mater. 24, 3738-3745 (2012).

47. Chockla, A. M. et al. Silicon Nanowire Fabric as a Lithium Ion Battery Electrode Material. J. Am. Chem. Soc. 133, 20914-20921 (2011).
48. Klavetter, K. C. et al. A high-rate germanium-particle slurry cast Li-ion anode with high Coulombic efficiency and long cycle life. J. Power Sources 238, 123-136 (2013).

49. Palacin, M. R. Recent advances in rechargeable battery materials: a chemist's perspective. Chem. Soc. Rev. 38, 2565-2575 (2009).

50. Beattie, S. D., Larcher, D., Morcrette, M., Simon, B. \& Tarascon, J. M. Si electrodes for Li-ion batteries - a new way to look at an old problem. J. Electrochem. Soc. 155 , 158-163 (2008)

51. Kovalenko, I. et al. A Major Constituent of Brown Algae for Use in High-Capacity Li-Ion Batteries. Science 333, 75-79 (2011).

52. Darwiche, A. et al. Better cycling performances of bulk Sb in Na-ion batteries compared to Li-ion systems: an unexpected electrochemical mechanism. J. Am. Chem. Soc. 134, 20805-20811 (2012).

53. He, M., Kravchyk, K., Walter, M. \& Kovalenko, M. V. Monodisperse antimony nanocrystals for high-rate $\mathrm{Li}$-ion and $\mathrm{Na}$-ion battery anodes: nano versus bulk Nano Lett. 14, 1255-1262 (2014).

54. Kravchyk, K. et al. Monodisperse and Inorganically Capped $\mathrm{Sn}$ and $\mathrm{Sn} / \mathrm{SnO} 2$ Nanocrystals for High-Performance Li-Ion Battery Anodes. J. Am. Chem. Soc 135, 4199-4202 (2013)

55. Heß, M. \& Novák, P. Shrinking annuli mechanism and stage-dependent rate capability of thin-layer graphite electrodes for lithium-ion batteries. Electrochim. Acta 106, 149-158 (2013).

56. Nakahara, K., Nakajima, R., Matsushima, T. \& Majima, H. Preparation of particulate $\mathrm{Li}_{4} \mathrm{Ti}_{5} \mathrm{O}_{12}$ having excellent characteristics as an electrode active material for power storage cells. J. Power Sources 117, 131-136 (2003).

57. Park, C.-M., Kim, J.-H., Kim, H. \& Sohn, H.-J. Li-alloy based anode materials for Li secondary batteries. Chem. Soc. Rev. 39, 3115-3141 (2010).

58. Guo, H. et al. Copper nanowires as fully transparent conductive electrodes. Sci. Rep. 3, 2323; DOI: 2310.1038/srep02323 (2013).

59. Li, S., Chen, Y., Huang, L. \& Pan, D. Large-Scale Synthesis of Well-Dispersed Copper Nanowires in an Electric Pressure Cooker and Their Application in Transparent and Conductive Networks. Inorg. Chem. 53, 4440-4444 (2014).

60. Bodnarchuk, M. I., Kravchyk, K. V., Krumeich, F., Wang, S. \& Kovalenko, M. V. Colloidal tin-germanium nanorods and their Li-ion storage properties. ACS Nano 8, 2360-2368 (2014).

61. Zhang, M. et al. Large-scale synthesis of antimony nanobelt bundles. J. Cryst. Growth 268, 215-221 (2004).

62. Kieslich, G., Birkel, C. S., Stewart, A., Kolb, U. \& Tremel, W. Solution synthesis of nanoparticular binary transition metal antimonides. Inorg. Chem. 50, 6938-6943 (2011).

63. Wang, Y. W. et al. Antimony nanowires self-assembled from Sb nanoparticles. J. Phys. Chem. B 108, 16723-16726 (2004).

\section{Acknowledgments}

This work was financially supported by ETH Zürich (Grant Nr. ETH-56 12-2), the Swiss Federal Commission for Technology and Innovation (CTI-Project Nr. 14698.2 PFIW-IW) and by CTI Swiss Competence Centers for Energy Research (SCCER Heat and Electricity Storage). Electron microscopy was performed at Empa Electron Microscopy Center.

\section{Author contributions}

The manuscript was prepared through the contribution of all authors. M.W. carried out the synthesis and characterization of the materials and electrochemical measurements. R.E. performed STEM characterization. M.V.K. conceived and supervised this work, helped with analysis and co-wrote the manuscript.

\section{Additional information}

Supplementary information accompanies this paper at http://www.nature.com/ scientificreports

Competing financial interests: The authors declare no competing financial interests.

How to cite this article: Walter, M., Erni, R. \& Kovalenko, M.V. Inexpensive Antimony Nanocrystals and Their Composites with Red Phosphorus as High-Performance Anode Materials for Na-ion Batteries. Sci. Rep. 5, 8418; DOI:10.1038/srep08418 (2015)

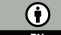

This work is licensed under a Creative Commons Attribution 4.0 International License. The images or other third party material in this article are included in the article's Creative Commons license, unless indicated otherwise in the credit line; if the material is not included under the Creative Commons license, users will need to obtain permission from the license holder in order to reproduce the material. To view a copy of this license, visit http://creativecommons.org/licenses/by/4.0/ 